\title{
A Novel Brain Computer Interface on its types on a future look
}

\author{
${ }^{1}$ Subha Austalekshmi.T.V, ${ }^{2}$ Vijaya Sarathi.S \\ 1Asst Professor, Department of Electronics\& Control, Sathyabama University, Chennai, TamilNadu, INDIA.. \\ 2Asst Professor, Department of Electronics \& Electrical engg., Indira Institute of Technology, Chennai,
} TamilNadu, INDIA..

\begin{abstract}
A Brain computer interface acts as communication channel between human brain and the computer system. Many challenging disorders and diseases is been faced by the brain in the past, but still that may not be the case in the mere future. Among all the challenges, paralysis stands the most. An advanced brain computer interface called as Nano brain computer interface that could provide a two - way communication between human brain and external system. The major goal of BCI research is to develop a system that allows disabled people to communicate with other persons and help in replacing the destroyed neurons. This paper provides an insight into the future look BCI, its application recent developments and an open discussion in the measures and evaluation of brain activity.
\end{abstract}

Key Words: Nano BCI, BCI, Invasive and Non-Invasive, EEG, MEG, MRI and FRMI.

\section{Introduction:}

Hans Berger's was the inventor of EEG that is used to record the signal from the human brain for the first time in 1924. It was too complicated to record the signals of the brain, but still Mr. Berger's was able to record his first signal such as the alpha wave $(8-12 \mathrm{~Hz})$ of the oscillatory activity in the brain, after which it was named as Berger's wave. The development required to insert silver wires under the scalp of the patients for the recordable work of the signal, which was later modified as silver foils attached to the head of the patients with the help of a rubber bandage around the area of investigation. EEG was the former instrument used in analyzing the interrelation of the wave diagrams of the brain that permitted to research the activities of the brain.

Our brain, spinal cord and peripheral nerves make up a complex network for work, integrated information - processing and control system known as central nervous system. They regulate all the conscious and unconscious facts of one's life. Neuron plays an important role in constituting this so called brain for different performance in everyone's life.

As we know our brain is made of approximately 80-120 billion nerve cells called Neurons. Neurons posses the amazing power to receive and transmit electro chemical signals which is related as gates and wire as such of a computer.

Our sensory organs movement is controlled by the communication transferred between the nervous system and the central nervous system which wallus in hand with the brain and spinal cord. Disturbed communication of the nerve impulses anywhere on the pathway from the brain to the peripheral muscles can corrupt the control of our muscle movement and thus leading to a weakness condition or loss of control or loss of co- ordination support that in turn leads to paralysis.

As of now we have few assisting equipments and technologies which help in such situation. Still there are technology helps in studying the structural design of brain, the active participation of brain, emotion and feel of the brain control etc;

\section{Development over the years:}

Electroencephalography stands as the head master for an excellent temporal resolution provider. EEG recordings are the most thoroughly studied technology used in interfacing the system of requirement and have the advantage over the others. a technique for mapping the activities of the brain by using the magnetic fields generated by electrical currents occurring naturally. MEG includes basic applications such as localizing regions affected by pathology before surgery, determining the functional structure of the brain. Human can reported one -dimensional MRI image in 1950. On further development, Damadiancuated the world's first magnetic resonance imaging machine in 1972. Damadian along with Larry Minlcoff and Micheal goldsmith, subsequently took an effort to perform the first MRI body scan of a human being on july 1977 , as of now many research division have taken interest towards the development of MRI. Diffusion MRI measures the diffusion of water molecules in biological tissues. Diffusions MRI is useful for the diagnosis of conditions like stroke or neurological / disorders that helps in diagnosis the connectivity of white matter axons in the central nervous system. Diffusion tensor imaging enables the researches to make brain maps of fiber that show the direction to examine the connectivity of different regions in the brain. 
The next level of image is diffusion weighted imaging. Then we have magnetization transfer on line. Magnetization transfer refers to the transfer of longitudinal magnetization from free water proton to hydration water proton in NMR and MRI. Finally functional MRI measures signal changes in the brain due to concentration of blood in brain that leads to change in neural activities.

\section{Generation Of Bci:}

In this new generation Nano BCI, the electrodes placed for the analysis of the brain activities are replaced by the nanobot bodies that constitute the work of recording the signal from the brain. This replaced nanobot can transmit and receive the signal require for a normal brain activity. In order to develop a new generation nano BCI, it is our duty to have a thorough understanding about the present BCI its types, hardware and software implementation of the system.

Majorly scientist and researchers talk about three important types of BCI Invasive, partially invasive and Non - invasive brain computer interfaces.

Invasive are those which are implanted directly inside the brain to record the highest quality of signals that provides functionality to paralyzed people. As the electrode is rested in the grey matter, it helps in restoring the vision as well as the use of limbs by using brain controlled robotic arms and legs. Since they made to rest in the grey matter, the electrodes reacts to a foreign object in the brain, thus causing the signal to become weaker or lost. People do not show any interest towards this type of BCI as it makes them scary as the insistent they know the procedure of implementation.

Partially invasive brain computer interfaces are implemented inside the skull but rest outside the brain. This type has a less risk of scan tissue formation compared to invasive BCI.

Non-invasive BCI, which considered being very safest compared to other types. This technique is one in which medical device or scanning device is mounted on caps or headbands that read brain signals. The most popular devices under this type BCI are the EEG which is cheap, easy to use and portable.

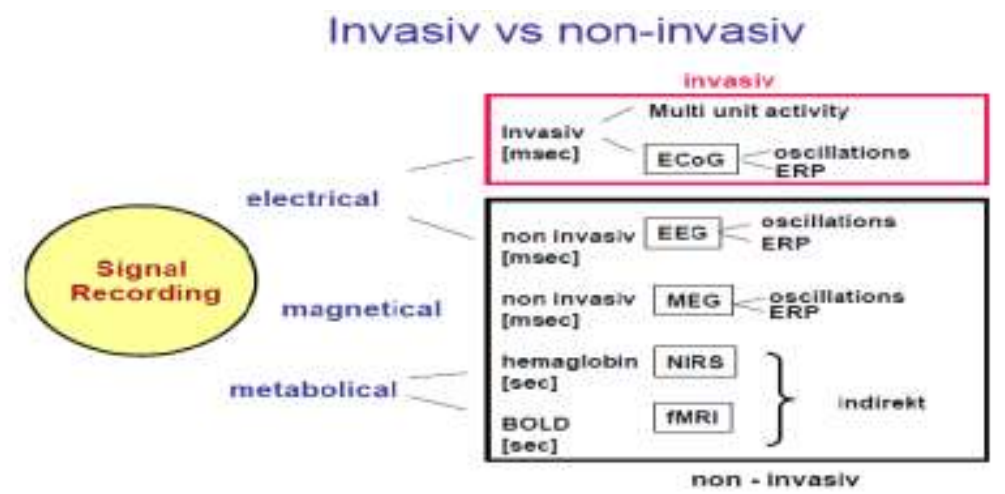

Basic Classification Of Bci:
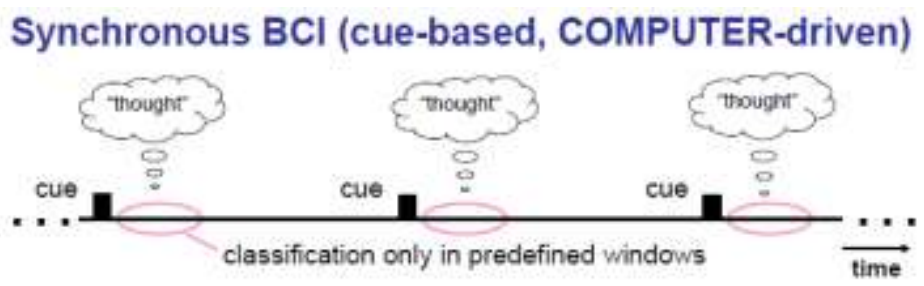

\section{Asynchronous $\mathrm{BCl}$ (uncued, USER-driven)}

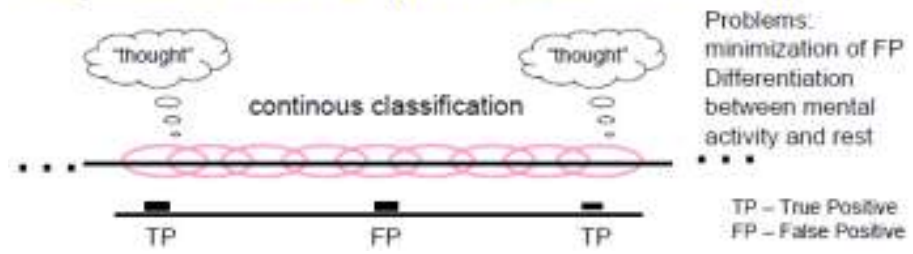

\section{Components Of Bci System:}

A BCI system consists of 5 sequential components 1) signal recording 2) convention 3) feature extraction 4) feature translation 5) device. 


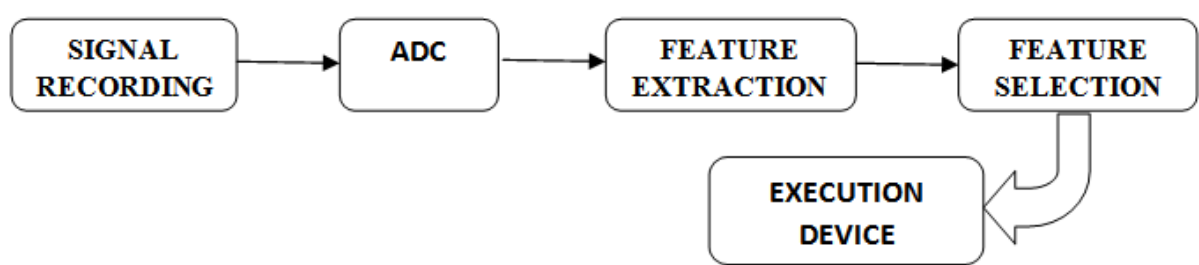

\section{Signal Recording And Convertion:}

The record signal from the brain is an electrical signal, after which it is converted into a digital form. This acquisition is called as data acquisition. Different parts of the brain are responsible for activity of various parts of the human body.

\section{Feature Extraction:}

This feature may be related to the shape of the time waveform analysis, to individual frequency analysis, to the power density spectrum, time frequency analysis, time frequency. Analysis, short time Fourier transform - STFT, wavelet transform - DWT, auto regressive models (AR) etc; mostly P300 potential is used to train the classifier.

\section{Feature Selection:}

Most difficult and important task of this system is said to be feature solution feature solution measure vary from day to day and even from section to section for the same user.

The best idea for this complexity to make ease only nanobots would help in build a unit within the brain to second, analyze, extract and selection and act. So in mere future we may end with nano BCI that will perform all modules of BCI that will perform all modules of BCI in a single unit.

\section{BCI-applications}

1. Spelling device/virtual keyboard (e.g. patients in a locked-in state)

2. Neuroprosthesis(e.g. tetraplegic patients)

3. Control of robotic arm/hand

4. Biofeedback therapy (e.g. reduction of epilepiic seizures,Enhanced stroke rehabilitation, treatment of attention deficit disorders)

5.Disabled persons to enable communication (Epidemiology of Amyotrophic lateral sclerosis:incidence1 2/100.000/year prevalence 5/100.000 patients (mainly male) older than 50 years or brain stem stroke, muscle dystrophies, ....also short term handicapped people)

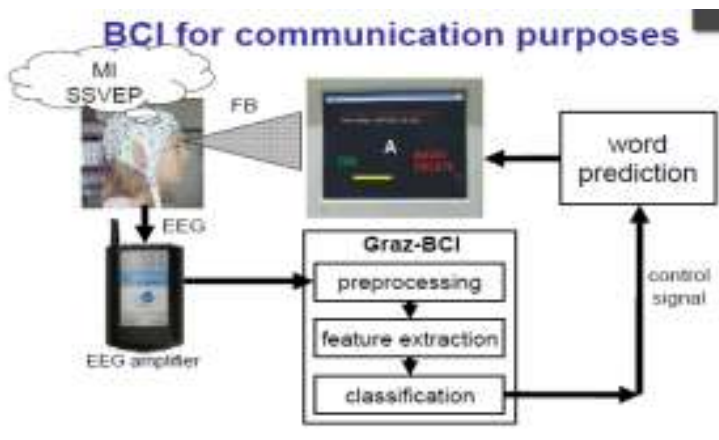

Future Goal:

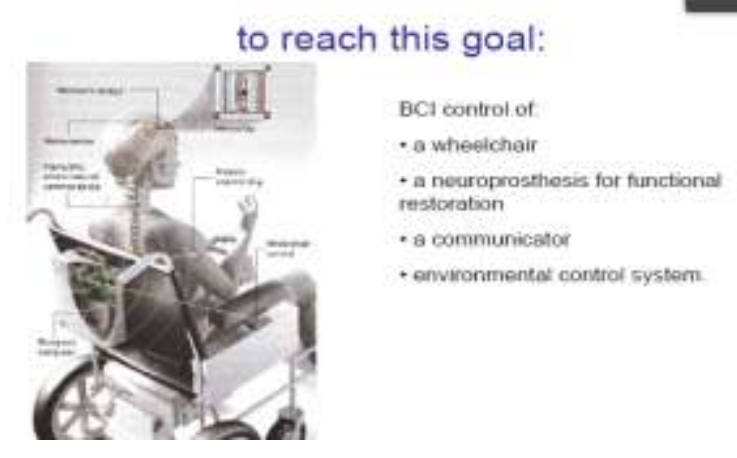




\section{A New Generation Nano Bci:}

The emerging of nanotechnology in the 1980s caused a great change the evolution of medical field. The experimental advances such as the invention of the scanning tunneling microscope was develop in 1981 and the discovery of fullerenes in 1985, with the education and popularization of a conceptual framework for the goals of nanotechnology beginning with the 1986 publication of the book called 'Engines creation'.

Nano technology stands for a engineering functional systems at the molecular scale. Brain -computer interfaces are still at its infamy were research continues into both brain functioning and the ways to map it effectively to electronic data. Many people are doing research into the field of BCIs that work by directly linking together an external device with a human brain. One way or a two way process still stands as theoretical work, but two way interfaces have not yet been successfully implemented. So for bidirectional communication nano can help the future for the disability to interface with their real external world.

\section{Conclusion:}

Nano BCI is still under the advancement of technology. The research in this area is rapidly increasing as the years move. Till date the basic principles have been studied. It may take a decade from now to the taste the fruits of nano by the common man in the real world.

\section{References}

[1]“ Edlinger, G., Holzner, C., Groenegress, C., Guger, C. and Slater, M. Goal-orientated control with braincomputer interface. Foundations of Augmented Cognition. Neuroergonomics and Operational Neuroscience, Springer (2009), 732-740.

[2] Pfurtscheller G et al.: Human Brain-Computer Interface, In: Riehle A., Vaadia E., MotorCortex in Voluntary Movements, pp. $367-$ 401, (2005), CRC press

[3] Dornhege, G, Millán, J., Hinterberger, T., McFarland, D.J., Müller, K.-R. Toward Brain-Computer Interfacing, MIT Press, Cambridge, MA, 2007.

[4] Allison, B.Z. and Pineda, J.A. Effects of SOA and flash pattern manipulations on ERPs, performance and preference: Implications for a BCI system. Int. J. of Psychophysiology, 59, 2 (2006), 127-140.

[5] Pfurtscheller et al.: 15 years of BCI research at Graz University of Technology:Current projects, IEEE TNSRE (2006), 14(2):205210 . 